\title{
Hiba Yūsuf
}

\section{Un flacon de naphte en verre émaillé de l'époque maml vke}

Le musée d'art islamique du Caire renferme parmi sa collection un vase sphéro-conique $^{(1)}$ en verre épais, transparent, de couleur jaunâtre, muni de bulles et de stries, soufflé dans le moule. Il porte le nom de l'émir

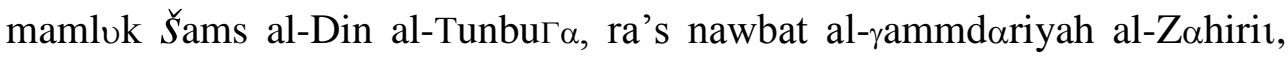
vers l'année 693 H./ 1293-1294 (Pls. I-VI). On y lit:

$$
\begin{aligned}
& \text { مما عمل برسم الجناب العالى/ المولوى الأميرى الكبيرى/ }
\end{aligned}
$$

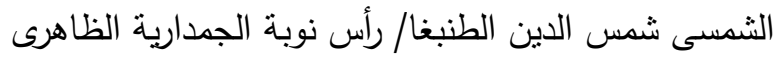

Il présente une panse piriforme, un goulot étroit et un culot pointu instable, de taille relativement grandiose, de $21.5 \mathrm{~cm}$. d'hauteur: l'étude comparative avec des objets similaires fabriqués en poterie (Pls. IX-X), démontre que leur hauteur varie, le plus souvent, entre $13 \mathrm{~cm}$. et $17 \mathrm{~cm}$.

L'ornementation, simple et raffinée, est exécutée en émail rouge et bleu, constituant un décor épigraphique, floral, animalier et armoiries (un blason composé, représentant un lion gravé au dessus d'un losange).

En voici les dimensions:

Hauteur totale: $21.5 \mathrm{~cm}$.

Hauteur du goulot: $5 \mathrm{~cm}$.

Diamètre de la panse (dans sa plus large partie): $54 \mathrm{~cm}$.

Diamètre de l'orifice: $9 \mathrm{~mm}$.

Il faudra noter à cet égard que des vases identiques, en verre et en poterie, ont été trouvés à Fus $\tau \alpha \tau$ et dans tous le Proche Orient ainsi qu'en Iraq, en Iran et en Afghanistan. ${ }^{(2)}$

(1) No. 18038 .

(2) A. Goulchani and C. Adle, A Sphero-Conical Vessel as Fuqa'a or a Gourd for "Beer", Muqarnas, 9, (1992), p. 72. 
Signalons à titre d'exemple un vase sphéro-conique en verre émaillé au nom du sultan rasulide al-Ašraf CUmar ibn Yusuf (694-696 H./ 12951296), conservé au musée du Louvre (Pl. VII). Le décor de l'épaule représente un aigle aux ailes déployées, répété trois fois, alternant avec un médaillon circulaire renfermant des motifs géométriques (des séries de triangles). En haut et en bas des médaillons, on constate des calices.

Le décor de la panse constitue un bandeau épigraphique en nashi mamluk qui lit:

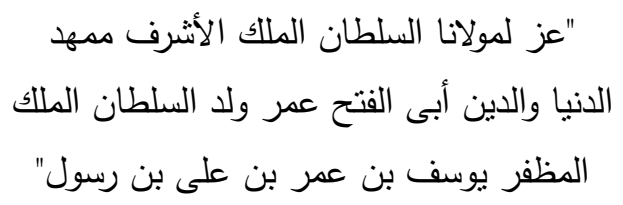

"Gloire à notre maître le sultan al-Malik al-Ašraf mumahid al-duniy $\alpha$ wa'l din, Abi al-Fatך 'Umar fils du sultan al-malik al-Muzaffar Yusuf ibn “Umar Ibn ‘Ali ibn Rasul”."(1)

Notons aussi, un vase sphéro-conique, en verre épais manganèse au nom du même sultan, conservé au musée du Victoria et Albert (Pl. VIII). Le décor de l'épaule constitue des calices trilobés ainsi que des médaillons circulaires renfermant des séries de triangles; le tout fut exécuté sur un fond de rosettes à six pétales ainsi que des motifs floraux variés. Le décor de la panse représente une inscription en nashi mamluk, sur un fond de rinceaux spiraux, qui lit:

"عز لمولانا السلطان الملك الأشرف أبو الفتح عمر ولد السلطان الملك المظفر"

"Gloire à notre maître le sultan al-Malik al-Ašraf Abu'l Fatn

'Umar fils du sultan al-malik al-Muzaffar". fois ${ }^{(2)}$

Le décor du culot comporte des calices trilobés répétés plusieurs

(1) Anmad ‘Abd al-Raziq, al-Funun al-islamiya fi'l caørayn al-ayyubi wa'l mamluki, Le Caire (2006), p. 264.

(2) Anmad 'Abd al-Raziq, al-Funun al-islamiya fi'l caørayn al-ayyubi wa'l mamluki, pp. 263-264. 
Le musée National du Kuwait renferme un exemple similaire (P1. IX) de l'époque fatimide (Ve/ XIe siècle) en poterie sans glaçure, aux parois épaisses, au décor moulé, exécuté en relief. ${ }^{(1)}$

Le musée d'art islamique du Caire renferme, à son tour, un grand nombre de vases sphéro-conique en poterie, avec ou sans glaçure, tel un vase en poterie, au décor moulé, sous glaçure bleue (Pl. X). Le décor de la panse et de l'épaule constitue une série de losanges, exécutés en reliefs.

La destination de ces objets est encore regardée comme douteuse: leur fonction reste encore sujet à controverses, et les archéologues hésitent entre plusieurs explications: des éolipiles, des containers de liquides précieux tel le mercure, des flacons de parfum, des gourdes de vin ou de bière (fuq $\alpha^{c}$ ), des étalons de poids, des lampes à huile ou des flacons de naphte.

Certains auteurs considèrent ces vases comme des éolipiles. ${ }^{(2)}$ L'éolipile; la "balle d'Eole"(3) était décrite comme un récipient plus ou moins sphérique, muni d'un pertuis très étroit. A demi rempli d'eau et placée dans le foyer, elle souffle un violent jet de vapeur qui attise le feu. ${ }^{(4)}$

Vis-à-vis de ces auteurs, cette interprétation évite tous les obstacles que soulevaient les autres. ${ }^{(5)}$ D'après Seyrig: 'l'étroitesse du goulot n'est pas seulement nécessaire pour assurer la violence de jet de vapeur; elle cesse également d'entraver le remplissage, car il suffisait de chauffer fortement le vase à sec, puis de le plonger dans l'eau, pour que celle-ci fut aspirée à l'intérieur par suite de refroidissement. L'épaisseur des parois se justifie par la double nécessité d'aller au feu et de subir la pression de la

(1) Ghada Qaddoumi, La variété dans l'unité, Dar al-ątar al-islamiya, Kuwait, (1987), p. 50 .

(2) W. L. Hildburgh, "Aeolipiles as Fire Blowers", Archaeologia, (Oxford), XCIV (1951), pp. 27-55; Henri Seyrig, Flacons? Grenades? Eolipiles? Syria, XXXVI, Paris, (1959), pp. 81-89.

(3) Repris du latin Aeolus, gr. Aiolis, dieu des vents.

(4) Henri Seyrig, Flacons ?, pp. 87-88.

(5) Supra. 
vapeur; leur porosité n'a plus d'inconvénient, et l'absence des restes d'un contenu s'explique toute seule". ${ }^{(1)}$

D'ailleurs, Ettinghausen remarque que tous les éolipiles connus en Europe et en Himalaya-excepté un seul-étaient fabriqués en métal; de plus, aucun des objets concernés ne porte des signes d'avoir approché le feu et, les textes arabes, à leur tour, ne comportent aucune information concernant l'usage d'ustensiles aussi banal à l'époque. ${ }^{(2)}$

D'autre part, il est assez évident que cette hypothèse était axée, en particulier, sur les exemplaires fabriqués en poterie et en céramique, en excluant ainsi les exemples similaires en verre, y compris celui faisant l'objet de notre étude. Les vases en question ne sont donc pas, selon toute apparence, des éolipiles.

D'autres voient dans ces vases des containers destinés à la conservation de liquides précieux tels le mercure et les drogues, destinés à être commercialisés et transportés: ${ }^{(3)}$ ils signalent l'existence des traces de mercure sur la face intérieure de très peu d'exemples. L'épaisseur des parois ainsi que le fond pointu leur paraissent convenables pour la conservation d'un liquide aussi visqueux que le mercure; quant à l'étroitesse du goulot, elle permet une fermeture facile. ${ }^{(4)}$

Pour argumenter leur hypothèse, les auteurs font allusion à un usage extensif de mercure dans la préparation de drogues et de cosmétiques. ${ }^{(5)}$

(1) Henri Seyrig, Flacons? p. 88.

(2) R. Ettinghausen, The Uses of Sphero-Conical Vessels in the Muslim East, Journal of Near Eastern Studies, Chicago, XXIV, (1965), p. 225; A. Goulchani and C. Adle, A Sphero-Conical Vessel as Fuqa'a or a Gourd for "Beer", p. 73.

(3) Greville J. Chester, "On the Pottery and Glass Found in the Excavations", The Recovery of Jerusalem, A Narrative of Exploration and Discovery in the City and the Holy Land, London, (1871), pp. 480-481; R.Ettinghausen, Sphero-Conical Vessels, pp. 218-223; Stéphane Pradines, Benjamin Michaudel et Julie Monchamp, La muraille ayyubide du Caire: les fouilles archéologiques de $\mathrm{B} \alpha \mathrm{b}$ al-Barqiya et $\mathrm{B} \alpha \mathrm{b}$ al-Manruq, AnIsl., XXXVI, Le Caire, (2002), p. 309.

(4) R. Ettinghausen, Sphero-Conical Vessels, p. 222.

(5) Voir 1' Appendice fait par S. D. Goitin dans Sphero Conical Vessels, p. 229. 


\section{Pourtant, deux difficultés surgissent à nouveau:}

La première difficulté porte sur les innombrables exemplaires trouvés dans tout le proche orient; un nombre qui parait quand même douteux pour l'usage d'une matière aussi précieuse et rare tel le mercure.

La deuxième difficulté comprend l'existence des vases similaires (ou presque) fabriqués en verre: la finesse des parois tout de même la fragilité du verre ne permet pas un usage approprié de containers pour les liquides précieux. $^{(1)}$

Pourrions nous donc conclure que cette hypothèse, ne peut s'appliquer, en tout état de cause, qu'à un nombre très restreint d'exemplaires?

Ettinghausen, à son tour, et en se basant sur un relief en stuc trouvé dans le palais ummayade "Qaఠr al-Hir al-Garbi", représentant une femme debout et tenant un flacon similaire dans sa main, a suggéré l'usage d'un flacon de parfum. ${ }^{(2)}$ Cependant, nous avons constaté que le goulot du flacon est assez évasé, et diffère donc de nos objets au goulot très étroit. D'autre part, il est impossible de les poser debout; faute d'un piédouche: Une simple comparaison entre ces objets et les flacons de parfums connus à l'époque musulmane démontre une très grande différence typologique. ${ }^{(3)}$ Selon Sauvaget on aurait du mal à imaginer que les musulmans, auxquels leurs propres potiers et les importations de Chine fournissaient des céramiques si délicates, aient jamais enfermé des parfums, dans des vases aussi lourds, aussi instables, aussi mal aisés à manier, et aussi grossiers. ${ }^{(4)}$

(1) Ettinghausen, Sphero-Conical Vessels, pp. 223-224; Goulchani et Adel, A SpheroConical Vessel, p. 72.

(2) Ettinghausen, Sphero-Conical Vessels, p. 224; Voir aussi Lane, Early Islamic Pottery, London, (1947), pp. 27-28; Géza Fehérvari, Ceramics of the Islamic World, in the Tareq Ragab Museum, London, p. 208.

(3) Voir notre thèse de Master dactylographiée "La toilette féminine à l'époque fatimide en Egypte”, Faculté de Tourisme et d' Hôtellerie, Université de Hilwan, Le Caire, (1995), pp. 245-247, Pls. LIV/ a, b , LV/ a, b , LVI/ a, b.

(4) Sauvaget, Flacons à vin ou grenades à feu grégeois? Dans Mélanges Grégoire (Annuaire de l'Institut de Philologie et d'Histoire Orientales et Slaves), IX, Bruxelles, (1949), p. 526. 
Un autre groupe d'archéologues pensent qu'il s'agit des flacons destinés à la conservation de vin ou de bière voir des gourdes. ${ }^{(1)}$ Mais les difficultés pratiques s'y opposent à nouveau. D'après Seyrig: "Les parois poreuses, très épaisses et donc très absorbante, ne faisaient pas un récipient de choix pour la très faible quantité de liquide que le vase était capable de renfermer. La forme du vase, impossible à poser, se prêtait mal à un usage de table et sa minuscule capacité ne s'accordait pas mieux avec la coutume des banquets. Le goulot, cet étroit pertuis dont le diamètre n'est parfois que de $3 \mathrm{~mm}$., et le plus souvent de 4 à $5 \mathrm{~mm}$., se refuse à toute versée commode $\mathrm{du}$ contenu. Ce même goulot, d'ailleurs, entrave par son exiguïté le remplissage, qui pourtant devait se faire en masse". (2) Ces arguments, déjà signalés, excluent à leur tour l'usage de flacons à vins.

D'ailleurs on devrait rapporter que Goulchani et Adel avaient lu, sur certains exemplaires trouvés en Azerbaïdjan et en Iran, des invocations liées à une boisson saine اشرب هنيا; ils pensent ainsi qu'il s'agit de fuq $\alpha$ 'a, kuzi fuq $\alpha^{c}$, ou bien gourde de bière. ${ }^{(3)}$

En revanche, on devrait rappeler que la forme des dits objets, diffère plus au moins des nôtres; et nulle inscription similaire n'a été rencontrée sur les exemples trouvés en d'autres régions.

Qu'il nous soit permis de référer ici sur le thème de "la variété dans l'unité" qui met en évidence la variété régionale dans l'art islamique. Rappelons que les tendances à disséminer, cataloguer et classifier les objets selon le lieu et la période de production, sont communes parmi les islamologues. Les objets de chaque région sont empreints de caractéristiques propres aux régions où ils sont produits; en faisant ainsi, chaque région a contribué d'une manière marquante à la création d'une tradition islamique artistique.

(1) Ghada Qaddoumi, La variété dans l'unité, p. 50; Goulchani et Adel, A Sphero-Conical Vessel, pp. 72-92.

(2) Henri Seyrig, Flacons?, p. 83.

(3) Goulchani et Adel, A Sphero-Conical Vessel, p. 74. 
La nature de ces contributions est toutefois déterminée par plusieurs facteurs. ${ }^{(1)}$

A la lumière de cette considération, pourrions nous supposer qu'il s'agirait donc ici d'un usage régional de vases sphéro-conique?

Les fouilles d'al-Fus $\tau \alpha \tau$ dirigées par le musée d'art islamique du Caire ont mis au jour quelques exemplaires renfermant, les uns, des traces de naphte et, les autres, des mèches au goulot. ${ }^{(2)}$ Ces trouvailles entraînèrent une nouvelle hésitation entre lampes à huile ou qwarir naf $\tau$, flacons à feu grégeois.

D'ailleurs, on doit rappeler que l'étroitesse de l'ouverture ne permettait pas une bonne oxygénation de la flamme et on note une absence systémati-que de noir de fumée sur le goulot. ${ }^{(3)} \mathrm{D}$ 'autre part, le fond pointu instable et l'absence de toutes sortes d'anses ne permettaient pas un usage pratique; ce qui exclut, à son tour, leur utilisation en tant que luminaires.

Reste donc l'hypothèse la plus probable de "qarvrat naf $\tau$ ", flacon à feu grégeois, qui trouve un fort appui parmi les archéologues. ${ }^{(4)}$

Ils trouvent que la forme ovoïde (parfois aussi globulaire) de nos objets aux goulots étroits, s'adapte facilement à l'usage de flacons de naphte:

(1) Pour mieux de détails concernant ce sujet, voir Ghada Qaddoumi, La variété dans l'unité, pp. 11 et suivants.

(2) Munammad `Abd al-`Aziz Marzoq, al-Funun al-zuhrufiya al-islamiya fi miør qabl alfarimiyyin, Le Caire, (1974), pp. 174-175

(3) Stéphanes Pradines, Les murailles, p. 308.

(4) Ali Bahgat et Félix Massoul, La céramique musulmane de l'Egypte, Le Caire, (1930), p. 89; Sauvaget, Flacons à vin?, pp. 525-530; M. Mercier, Le feu grégeois, les feux de guerre depuis l'antiquité, la poudre à canon, Paris, (1952), pp. 153-158; Ernest Kuhnel, Islamic Arts, London, (1963), p. 125; 'Abd al-Ra'uf 'Ali Yusuf, Dirasa fi'l fuhor al-

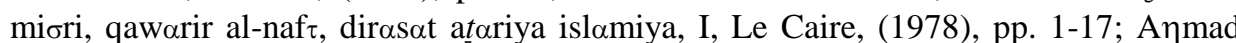
'Abd al-Raziq, al-Hadara al-islamiya fi'l cuбur al-wus $\tau \alpha$, Le Caire, (1990), p. 202; al_ayš al-miøri fi'l caør al-mamluki, Le Caire, p. 143; al-Funun al-isl $\alpha$ miya $\eta$ at $\alpha$ nihoyat alcaor al-farimi, Le Caire, (2001), p. 159-161; ‘Abd al- 'Aziz Salon Solim, al-Funun alislamiya fi'l caor al-ayyubi, Le Caire (2000), 41. 
L'absence de piédouche se justifie par le fait que ces objets n'avaient pas à être posés debout: on les entassaient les uns sur les autres, comme des boulets de canon, sur le sol d'un magasin ou d'un parc. ${ }^{(1)}$

Ces vases présentaient dans leur forme ovoïde, à peu près gros comme le poing, des aspérités émoussées afin d'être bien tenus en main, se prêtant ainsi à une projection facile. 'Abd al-Ra' uf 'Ali Yusuf signale, en outre, que cette forme sphéro-conique au fond pointu permettait un lancement de main assez éloigné. ${ }^{(2)}$

Certains auteurs pensent aussi que la forme des pots dépendait moins de l'usage auquel ils étaient destinés que de leur mode de fabrication, prenant part d'une véritable industrie, produisant en masse. Le tour d'un galbe ovoïde exigeait du potier moins de travail qu'une sphère puisqu'il l'obtenait par une simple pression des mains sans avoir à pratiquer de retouches ultérieures. ${ }^{(3)}$

Quant au décor, il n'offrait aucun inconvénient: il était le plus souvent exécuté dans le moule ${ }^{(4)}$ et avait pour rôle d'affaiblir la parois pour un rendement plus facile à casser lors du lancement.

Nous devrions rappeler, à cet égard, que les orientalistes trouvent du mal à imaginer que les musulmans aient donné tant de soins à décorer ces "engins de mort": ils omettent en effet la mentalité de l'artiste musulman visée sur les perfection artistique ainsi que sur son amour du "Beau", quoiqu'elle soit la fonction de l'objet produit.

L'étroitesse du goulot, à son tour, formait une précaution indispensable pour que ces vases chargés de matières inflammables ne répandent pas trop facilement leur contenu en se renversant durant le transport. ${ }^{(5)}$

(1) Sauvaget, Flacons à vin?, p. 528.

(2) 'Abd al-Ra'vf 'Ali Yusuf, Dirasa fi'l fuh $\alpha$ r al-miori, pp. 1-2.

(3) Certains de ces flacons étaient moulés, mais l'immense majorité a été faite au tour, Sauvaget, Flacons à vin? p. 528.

(4) Ernest Kuhnel, Islamic Arts, p. 125; ‘Abd al-Ra'uf ‘Ali Yusuf, Dirasa fi'l fuhar al-miøri, pp. 2-3.

(5) Sauvaget, Flacons à vin? p. 528. 
Cette étroitesse fut, en effet, mentionnée dans les manuscrits de

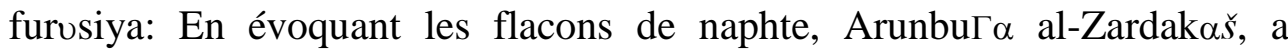
précisé, entre autre, l'usage de récipients de matières incendiaires, au goulot étroit. Il dit:

$$
\text { "تأخذ كراز فخار ضيق الرأس ويبيضه ويملأه ..."(1) }
$$

"Tu prends une cruche à goulot étroit ${ }^{(2)}$, tu blanchis et tu remplis ...".

A ce propos, nous devrions mettre en considération que les recettes variées de naphte ne comprenaient pas nécessairement du naphte visqueux, et, nous avons trouvé dans la littérature arabe quelques récits faisant allusion à la préparation de naphte volatil (permettant ainsi un remplissage facile). Nous signalons les récits suivants à titre d'exemple: En évoquant le siège de Saint Jean d'Acre et les incendies des tours en 586 H./ 1190, l'auteur de kitab al-rawdatayn nous apprend que le messager du calife abbasside alNarir (575-623 H./ 1179-1226) arriva au secours des troupes musulmanes. On y lit:

$$
\text { "ووصل و معه حملان من النفط الطيار ..."(3) }
$$

"... il arriva, accompagné de deux charges du naphte volatile"

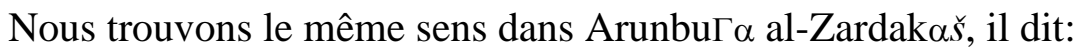

$$
\text { "تأخذ قطعة "تملأها نفط طيار ..." }
$$

"Tu prends une pièce que tu remplis avec du naphte volatil...".

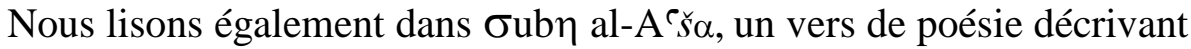
Miør:

$$
\text { "زحف عليها بعسكره الجرار و نفط مائه الطيار ..."(5) "(50) }
$$

"Il rampa sur l'Egypte avec son armée considérable et son naphte volatil ..."

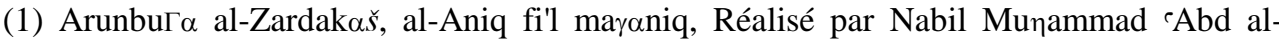
'Aziz, Le Caire, (1981), pp. 127-128.

(2) Dozy, Supplément aux dictionnaires arabes, Leyde, (1881), II, p. 455.

(3) Ših $\alpha$ b al-Din al-Maqdisi, Kit $\alpha$ b al-Rawdatayn fi ahbor al-dawlatayn al-nuriya wa'l

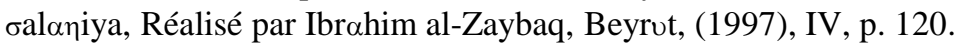

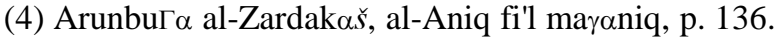

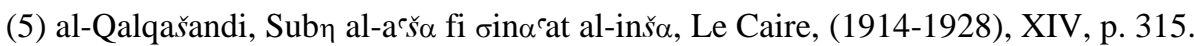


Les manuscrits de furusiya renferment, en outre, bon nombre de détails concernant les différents types de flacons de naphte: ainsi il en était de forme globulaire ou ovoïde, de taille moyenne ou grande (pour les mangonneaux), en poterie mais aussi en verre. ${ }^{(1)}$

Ces manuscrits nous fournissent également d'illustrations originales représentant des exemplaires variés de flacons de naphte y compris nos objets de forme sphéro - conique (Pls. XI-XIII).

Ces illustrations mettent donc fin à plusieurs intrigues concernant l'usage de ces récipients de forme inhabituelle, formant ainsi une preuve évidente de leur usage en tant que "flacons de naphte".

Quoi qu'il en soit, nous pouvons ajouter que l'usage des pots à feu grégeois se targue aussi sur l'appui des textes arabes, d'où il ressort que les projectiles à naphte étaient souvent des vases de terre et de verre; que ces vases étaient lancés sur l'ennemi par la main ou par des mangonneaux.

Nous rapportons les passages suivants à titre d'exemple.

L'historien al-Dahabi, auteur d'al--Ibar fi habar man Гabar, nous apprend qu'au cours de l'an 552 H./ 1157, Munammad $\breve{S}$ h fils du sultan

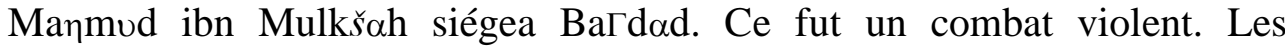
verriers produisirent pour le calife abbasside al-Muktafi (530-555 H./ 11361160), dix huit mille flacons de naphte:

"... حتي انه من جملة ما عمل له بعض الزجاجين ثمانى عشرة ألف قارورة للنفط" (2)

"Le même récit fut également confirmé dans al-Muntazim d' Ibn al-_awzi. On y lit:

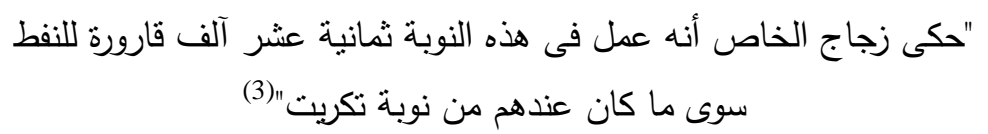

"Le verrier privé rapporta qu'il avait produit dix huit mille

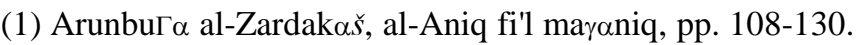

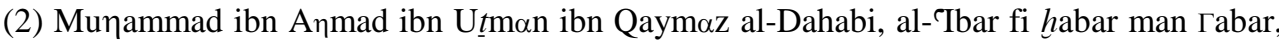

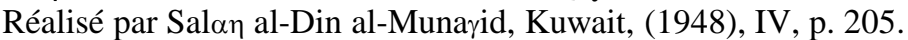

(3) Ibn al-_awzi, al-Muntazim min 257 H., Beyrut, (1358 H)., X, p. 171. 
flacons de naphte pendant cette invasion, hormis ce qui restait déjà de la bataille de Takrit".

Ces deux textes sont, en effet, d'une importance exceptionnelle puisqu'ils mettent en évidence l'usage de quantité innombrable de flacons de naphte fabriqués en verre.

Al-Maqrizi, à son tour, en évoquant l'incendie d'al-Fus $\tau \alpha \tau$ en 564 H./ 1168, dit:

"Ce fut un flot impétueux. Il semblait que les gens sortissent de leur tombeaux pour le jugement: le père ne se souciait pas de son enfant, le frère ne s'intéressait pas à son frère. On allait jusqu'à louer une monture de Misr à al-Qahira pour une somme de vingt dinars; puis $\breve{S} \alpha$ war fit envoyer à Misr vingt mille flacons de naphte et dix mille torche:

$$
\text { أرسل شاور إلى مصر عشرين ألف قارورة نفط وعشرة آلاف مشعل }
$$

Le tout fut réparti dans la ville, et la flamme et la fumée de

L'incendie s'élevèrent dans le ciel. Ce fut un effrayant spectacle".(1)

Ce récit était confirmé par la trouvaille d'un bon nombre de ces objets lors des fouilles d'al-Fus $\tau \alpha \tau$, comprenant nombre de pièces intactes. Nous avons déjà signalé que certains de ces flacons renfermaient des traces de naphte tandis que certains autres, avaient encore des mèches au goulot.

De tout ce qui précède, il nous est permis de déduire que l'usage de naphte-feu romain-était bien connu tout au long de l'époque musulmane, et eut son plus grand éclat à l'époque ayyubide et au début de l'époque mamluke.

C'est lorsque le feu grégeois perfectionné fut utilisé par les byzantins, alors que les arabes assiégeaient Constantinople en 50 H./ 670, la révolution de la guerre navale était achevée; l'eperonnage avait été abandonné et remplacé par un usage extensif de machine de jet de pierres, auxquelles le

(1) al-Maqdisi, Kit $\alpha$ b al-rawdatayn, II, p. 106; al-Maqrizi, al-Mawaciz wa'l Irtibor bi dikr

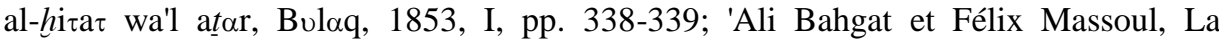
céramique musulmane, p. 89 , note 1 . 
jet de feu fut ajouté. L'emploi du feu grégeois en 50 H./ 670, faisait donc d'un nouvel armement important. ${ }^{(1)}$

De nombre de passages tirés de sources arabes concernant l'emploi du naphte, il nous apparaît qu'au milieu du Ie/ VIIe siècle, les musulmans utilisaient le naphte et qu'ils en employèrent de plus en plus avec le temps. ${ }^{(2)}$

D'après l'historien al-Suyuti, le naphte, feu grégeois, fut utilisé par les armées abbassides. ${ }^{(3)}$

L'examen de certaines sources arabes, révèle qu'il existait sans aucun doute une grande variété de mixtures incendiaires dont le nombre grandit régulièrement.

Nous signalons les compositions suivantes, d'après "Traité d'armurerie de Saladin”, de Murd $\alpha$ Ibn ‘Ali al- Tarsusi, ${ }^{(4)}$ à titre d'exemple:

* Description d'un naf $\tau$ artificiel puissamment incendiaire et atteignant tout ce que sur quoi les naphtes agissent:

Prends une cruche de bonne huile et une quantité de chaux vive que tu réduis en poudre, mélange-les en secouant fortement, et mets le mélange tout de suite dans une cornue à laquelle tu adaptes l'alambic. Prends une plaque, et allume en-dessous un feu vif, jusqu'à ce que le tout soit distillé. Prends l'huile distillée, ajoutes-y environ un douzième de nouvelle chaux vive pulvérisée comme précédemment et redistille de la même façon. Et avec ce qui a été distillé opère encore avec un seizième de chaux semblable

(1) Encyclopédie de l'Islam, Nouvelle Edition, VII, article "Naft", p. 886.

(2) Voir à titre d'exemple al-Tabari, Tarih al-umam wa'l muluk, Bayrut, (1407 H.), V, p. 643; Ibn al-_awzi, al-Muntazim, X, p. 169; al-Maqdisi, Kitab al-rawdatayn, IV, p. 120;

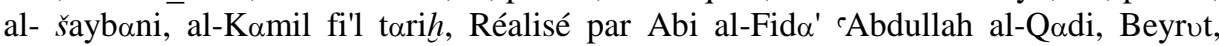
(1415 H./ 1995), X, p. 77; Ibn Katir, al-Bidayah wa'l nihayah, Beyrut, XII, pp. 320321; al-Dimašqay, šadarat al-dahab fi ahbor man dahab, Beyrut, S. D. , III, p. 241.

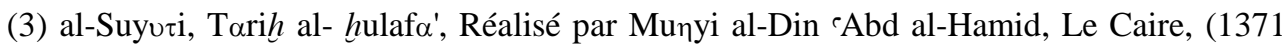
H./ 1952), 1, p. 299.

(4) Traité d'armurerie de Saladin, de Murda Ibn 'Ali al-Tarsusi, Syrie, Seconde moitié du VIe/ XIIe siècle, Oxford, Bodleian Library, University of Oxford-Voir Claude Cahen, Un traité d'armurerie composé pour Saladin, Bulletin d'Etudes Orientales, XII, Beyrut, (1948). 
et distille une troisième fois. Au produit ainsi obtenu ajoute à peu près un quart de naphte: il est propre à être employé, et il a une vertu incendiaire inégalée. ${ }^{(1)}$

* Fabrication d'un naf $\tau$ actif que l'on lance avec le mangonneau:

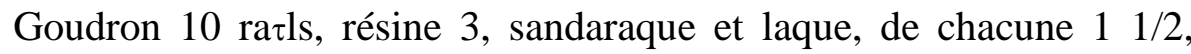
souffre pur de bonne qualité, débarrassé de toute terre, 3; graisse de rognons de chèvre dissoute, limpide, même quantité, fondre le goudron, jeter dessus ces graisses, puis la résine, après l'avoir complètement fondue; pulvérise intégralement les autres produits; revenir au produit cuit, mettre le feu et cuire jusqu'à ce le tout soit absolument unifié. Si tu veux tu veux t'en servir en temps de guerre, tu en prends une part, tu y ajoutes son dixième environ de soufre minérale, que l'on appelle naf $\tau$, de ce qui de lui tire sur le vert et ressemble à de la vieille huile; placer le tout dans une marmite; faire bouillir presque jusqu'à enflammer; prendre le vase avec un feutre. Alors lance le avec le mangonneau sur ce que tu veux: il le brûle sans jamais pouvoir être éteint. ${ }^{(2)}$

Les compositions déjà signalées et bien d'autres ${ }^{(3)}$ démontrent que diverses formules de naf $\tau$ se sont développées constamment.

Notons que pendant la plus grande partie de la période mamluke, le principal engin employé pour le lancement des projectiles au cours des sièges fut le man raniq, mangonneau. ${ }^{(4)}$

(1) Claude Cahen, Un traité d'armurerie composé pour Saladin, Bulletin d'Etudes Orientales, XII, Beyrut, (1948), p. 145.

(2) Claude Cahen, Un traité d'armurerie, p. 146.

(3) Pour plus de détail concernant ces compositions, voir Arunbu $\alpha$ al-Zardk $\alpha \breve{s}$, al-Aniq fi'l

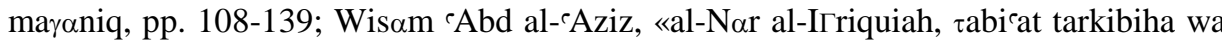

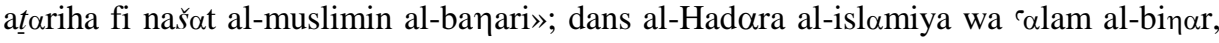
colloque organisé par la Fédération des historiens arabes, Le Caire, 6-8 Novembre, (1993), pp. 287-305.

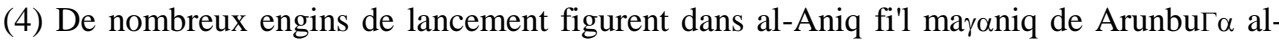

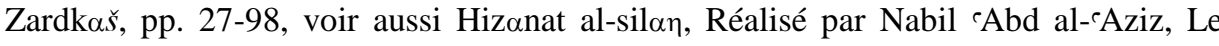
Caire, (1987). 
Quant aux lanceurs de naf $\tau$, ils étaient connus sous le nom de naff $\alpha$ in ou zarraqin. ${ }^{(1)}$

Les manuscrits de furusiya aussi bien que les textes arabes nous apprennent que ces naff $\alpha$ in étaient protégés par des vêtements spéciaux, les libas al-naff $\alpha$ iin (Pl. IX). Le passage suivant en est significatif:

"Prends des caparaçons doublés de feutre et en revêtir les chevaux après les avoir enduits d'un liquide résistant au feu; pour obtenir, prendre un ra $\tau$ d'amiante limoneuse, un de gomme arabique, quatre d'ocre rouge, deux de gypse, de farine blanche ce que tu veux, de blanc d'œuf ce que tu veux,

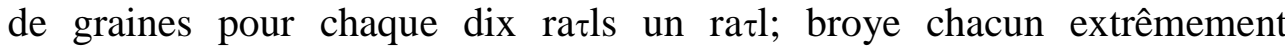
finement et tamise comme le Kohl; mêle le tout et pétris énergiquement avec du vinaigre de vin allongé d'eau assez pour n'être plus acide. Enduis de ce produit, copieusement, les caparaçons en question sur toute la surface. Vas alors prendre de la bourre choisie, susceptible de prendre la place des grelots et abreuve de naphte inflammable; enveloppe le fil de fer fin, et attache $\mathrm{s}$ en sur le devant et le derrière du cheval ainsi qu'autour de la croupe, le tout sur le caparaçon. Toi-même, revêts une cuirasse de grosse toile doublée de feutre et des pantalons de même matière, préalablement bien enduits de la pâte précédemment décrite, et place tout autour de la cuirasse, de ces grelots de bourre attachés à la cotte du cheval, ainsi que derrière son dos; sur la tête mets un bonnet de feutre enduit de la pâte susdite et à son sommet et autour place encore de ces grelots. Monte à cheval et prends garde qu'il ne sorte ni main ni pied". (2)

Les bur $\gamma \mathrm{s}$, tours d'offense ou de défense, offrant à leur tour une grande surface exposée, étaient volontiers revêtus de peaux ignifugées au vinaigre. $^{(3)}$

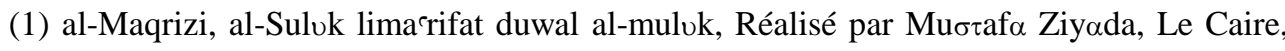

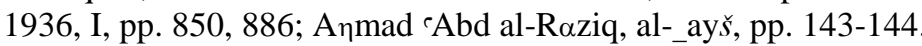

(2) Claude Cahen, Un traité d'armurerie, p. 147.

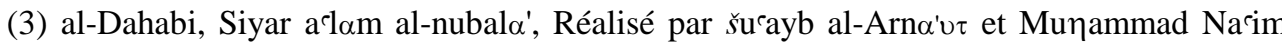
al--Irqsusi, Beyrut, (1413 H)., XXII, 210; Ibn Katir, al-Bid $\alpha y a h$ wa'l nihayah, Bayrut, XII, p. 335. 
C'est surtout à cause de divers moyens de se protéger des matières incendiaires, que l'emploi du naphte, tomba peu à peu en désuétude et déclina.

Un autre cause est que, les châteaux des croisées étant construits en pierre et contenant très peu de bois; l'efficacité de naphte comme arme offensive durant cette période, où l'initiative passa définitivement des Francs aux Musulmans, était devenu nulle. ${ }^{(1)}$

Par ailleurs, nous devrions mettre en considération que les flacons de naphte n'étaient pas tous destinés à la projection: l'étude des manuscrits de furusiya nous a permis de distinguer un autre type de naphte lancé avec les flèches. ${ }^{(2)}$ Ce naphte était en fait renversé sur les têtes des flèches et des lances pour leur donner des qualités incendiaires. D'après Arunbu $\Gamma \alpha$ alZardk $\alpha \breve{s}$, ces flèches étaient arrosées par le naphte des flacons. On y lit:

(3) "...... يحل بنفط القوارير ويرسم et ti

"... tu arroses avec le naphte des flacons et tu soudes"

Il serait donc admissible de penser que certains flacons-plus précisément ceux en verre émaillé-jouaient le rôle de "containers de naphte".

Etant donné que cette catégorie n'était pas destinée au bombardement, ceci expliquerait, peut-être, la trouvaille d'un bon nombre de flacons intacts, de plus le décor raffiné de certains d'autres.

A ce groupe, nous pourrions donc joindre notre flacon en verre émaillé conservé au musée d'art islamique du Caire, (ainsi que les deux autres exemplaires déjà signalés (Pls. VII-VIII).

Signalons que l'émail est une pâte vitreuse apte à la fusion avec d'autres matériaux afin de former une décoration à glaçure, résistantes et

(1) Sur son déclin et sa disparition complète voir David Ayalon, Gunpowder and Fire Arms in the Mamluk Kingdom, London, (1978), pp. 11-13.

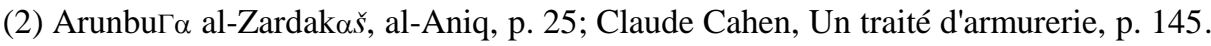

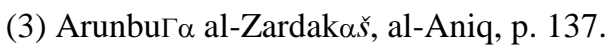


durables. ${ }^{(1)}$ Quant aux couleurs, ils s'obtiennent par des pigments métalliques composés d'oxydes mélangés à un matériel vitreux. ${ }^{(2)}$

Notons que la production prédominante de l'Egypte et de la Syrie durant la période mamluke était le verre émaillé et doré, produit dans les centres principaux de d'Alep, de Damas et du Caire. L'émaillage est considéré être un des accomplissements les plus raffinés dans la production du verre musulmans.

Qu'il nous soit permis de rappeler que cette technique colorée et déjà développée, a été transmise aux mamluks par leurs prédécesseurs, les Ayyubides. ${ }^{(3)}$

\section{La décoration du flacon est assez sobre et se résume ainsi:}

Le décor de l'étranglement du col: Un anneau circulaire de rinceaux très fin tracé en or.

Le décor de l'épaule: Il représente trois mandorles à arabesques aux extrémités fleuronnées alternant avec trois scènes d'oiseaux en combats, représentant un faucon attaquant sa proie: une oie.

Le décor de la panse: Un large ruban circulaire orné d'une inscription en réserve, cernée d'émail rouge sur un fond bleu. Il est interrompu par quatre médaillons circulaires, renfermant, chacun, un blason composé.

L'inscription est en nashi mamluk, caractères moyens, points nombreux. On y lit:

$$
\begin{aligned}
& \text { "مما عمل برسم الجناب العالى/ المولوى الأميرى الكبيرى/ }
\end{aligned}
$$

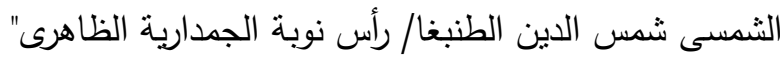

(1) Pour mieux de détails concernant les ingrédients de l'émail voir Erika Speel, Dictionary of Enamelling, (1998), p. 43.

(2) Concernant ces oxydes, voir C. Freestone et Colleen P. Stapleton, Composition and Technology of Islamic Enamelled Glass of the Thirteen and Fourteen Centuries, dans Gilded and Enamelled Glass from the Middle East, Edited by Rachel Word, London, (1998), pp. 122-128.

(3) Ghada Qaddoumi, La variété, p. 116. 
Voici qui a été fait pour son Excellence, notre maître, le grand émir, al- ̌̌amsi Šams al-Din al-Tunbu $\Gamma$, chef des jammdor, gardes robe, qui faisaient à tour de rôle leur service auprès du sultan al-Zahir (Baybars al-Bunduqdari)

Le texte précédent nous apprend que notre flacon a été fait pour $\breve{S}$ ams al-Din al-Tunbu $\alpha$, qui occupa le poste de Ra's nawbat al- $\gamma$ ammd $\alpha$ riyah, au cours du règne du sultan al-Zahir Baybars al-Bunduqdari (658-676 H. /1260-1277).

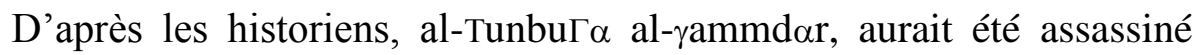
par ordre du sultan al-N $\alpha \sigma i r$ Munammad ibn Qal $\alpha$ won le 5 du mois de Safar 693 H./ Janvier 1294, suite à l'assassinat d'al-Ašraf Halil ibn Qalawon en 12 du mois d'al-Munarram 693 H./ Décembre 1293. Nous rapportons leur récit en suivant: "les mamluks du sultan al-Ašraf Halil amenèrent sept émirs, y

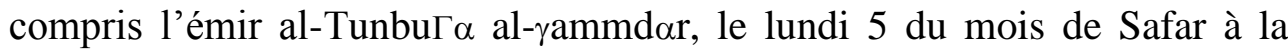

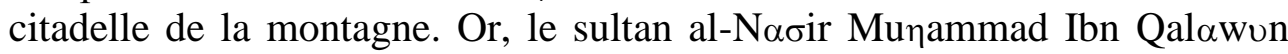
ordonna qu'on leur coupe les mains, ensuite ils firent cloués sur les chameaux, les mains dans les gorges. On les fit parader dans Misr et le Caire et demeurèrent ainsi jusqu'à la mort. Ensuite, ils furent récupérés par leurs familles et furent enterrés dans les cimetières". ${ }^{(1)}$ Le texte précédent nous permettrait donc de dater le flacon avant l'année 693 H./ 1293-1294. ${ }^{(2)}$

Les historiens soulignent que le titre $\gamma$ ammdar est l'abréviation du terme persan: $\gamma$ imi-d $\alpha \mathrm{r}^{(3)}$, ou $\gamma \alpha \mathrm{mah}-\mathrm{d} \alpha \mathrm{r} .{ }^{(4)}$ Le mot $\gamma \alpha \mathrm{mah}$ signifie robe (on a plus tard supprimer le alif pour faciliter la prononciation), et le mot $\mathrm{d} \alpha \mathrm{r}$

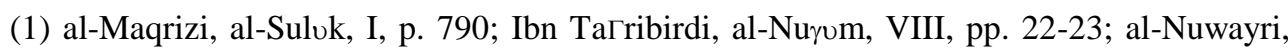
Nihayat al-arab fi funun al-adab, XXIX, fol. 304 v.

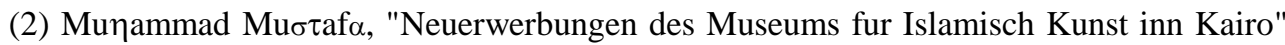
in Aus der Welt der islamischen Kunst, Festscrift fur Ernst Kuhnel, Berlin, 1959, pp. 92; 'Abd al-Ra'uf 'Ali Yusuf, Syro Egyptian Glass, Pottery and Wooden Vessels in Gilded and Enamelled Glass, p. 22.

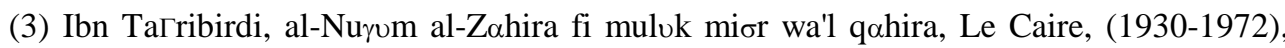
VII, p. 185.

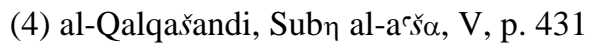


signifie celui qui tient ou celui qui porte. Ainsi ammdar signifie celui qui est responsable de la garde robe du sultan. ${ }^{(1)}$

On nous apprend, d'autre part, que les mamluks appelés $\gamma$ ammdars, étaient pour le rang et les fonctions, inférieur à ceux qui portaient le nom d'al-h $\alpha \sigma i k i y a h .{ }^{(2)}$

Quant au titre de ra's nawba, il était connu chez les tartares, et compta parmi les nouveaux rangs crées par le sultan al-Zahir Baybars alBunduqdari dans la hiérarchie mamlvke. ${ }^{(3)}$

Le mot نوبة nawba, signifie un relais, ce qui se fait à des intervalles réglées et successifs, et par suite, un corps de troupes qui, à tour de rôle, fait son service auprès du sultan, ou dans une place de guerre. ${ }^{(4)}$

Quant à ra's nawbat al- $\gamma$ ammd $\alpha$ riyah, il signifie le chef des $\gamma$ ammd $\alpha$, qui faisaient à tour de rôle leur service auprès du sultan, et compta parmi les harikiyah. ${ }^{(5)}$

Les historiens mamluks nous ont fourni les noms de plusieurs ra's

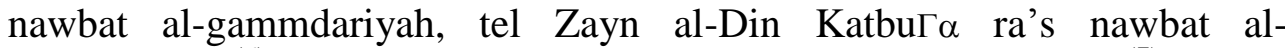
ammd $\alpha$ riyah $^{(6)}$, 'Izz al-Din Azdumur ra's nawbat al- $\gamma a m m d \alpha r i y a h^{(7)}, \operatorname{Ar} \Gamma u n$

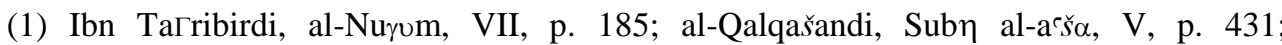
Quatremère, Histoire des sultans mamlouks, 1845, I, 1, p. 11, no. 11; Hasan al-B $\alpha \check{s} \alpha$, al-Funun al-isl $\alpha$ miya wa'l waz $\alpha$ 'if ‘al $\alpha$ al-atar al-carabiya, Le Caire, (1966-1967), I, pp. 356-357

(2) Quatremère, Histoire des sultans mamlouks, I, 1, p. 11, no. 11.

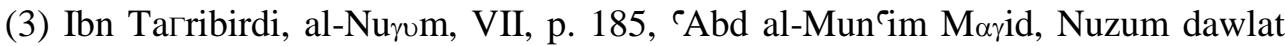
sal $\alpha$ in al-mamalik wa rusumihim fi mior, Le Caire, (1967), p. 48

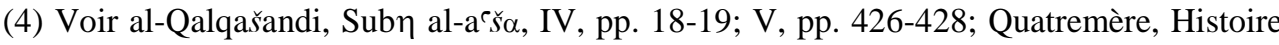
des sultans mamlouks, II, 1, p. 12 , no. 10.

(5) Quatremère, Histoire des sultans mamlouks, II, 1, p. 14.

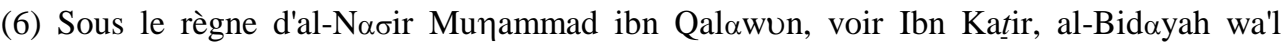
Nihoyah, XIV, p. 53.

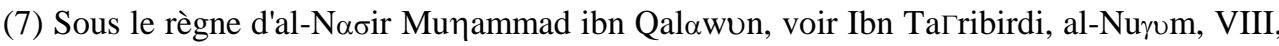
VIII, p. 177. 


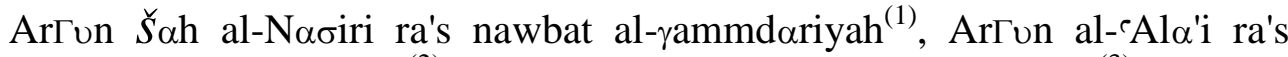

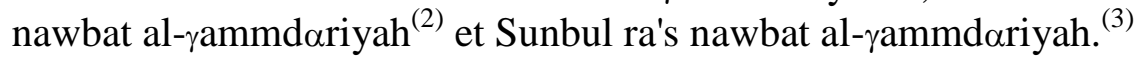

D'après Ibn $\breve{S} \alpha$ hin al-Zahiri, il existait à son époque sept ra's nawbat rammdariyah. ${ }^{(4)}$

Nous trouvons également ce titre sur d'autres objets d'art mamlvk, telle une pyxide en cuivre datant de l'an $700 \mathrm{H} . /$ 1300, et porte le nom de

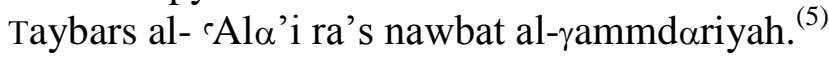

On le trouve aussi sur un encensoir daté du 741 H./ 1341, au nom de

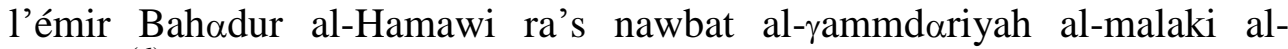
Naбiri. ${ }^{(6)}$

L'inscription susdite, est coupée par quatre médaillons circulaires, renfermant un blason composé. Il s'agit d'un lion gravé au dessus d'un losange (Pl. VI).

Qu'il nous soit permis de rappeler que les blasons ${ }^{(7)}$ ont constitué une partie assez importante du répertoire décoratif de l'art mamluk. Ces blasons étaient utilisés par les mamluks pour désigner le signe ou l'emblème

(1) Sous le règne d'al-Naбir Munammad ibn Qalawon, voir al-`Asqalani, al-Durar alKaminah fi a riyan al-ma'ah al-țamina, Réalisé par Munammad Han, Hidrabad, (1972), I, p. 415.

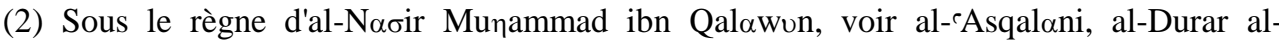
Kaminah, I, p. 419.

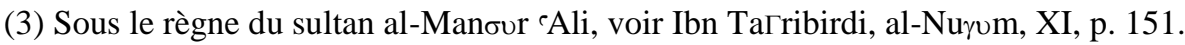

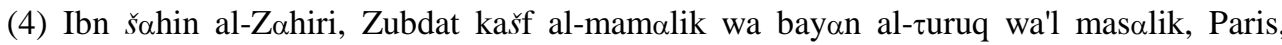
1894, p. 116; Hasan al-B $\alpha \check{s} \alpha$, al-Funun al-isl $\alpha$ miya wa'l waz $\alpha$ 'if, I, p. 357.

(5) Mayer, Saracenic Heraldry, A Survey, Oxford, (1933), p. 227; Hasan al-B $\alpha \check{\alpha} \alpha$, al-Funun Funun al-isl $\alpha$ miya wa'l waza'if, II, p. 550.

(6) Conservé au musée national de Florence, voir Mayer, Saracenic Heraldry, pp. 14, 95; Hasan al-B $\alpha \check{s} \alpha$, al-Funun al-isl $\alpha$ miya wa'l waz $\alpha$ 'if, II, p. 550.

(7) Le mot rank ou ran $\gamma$, blason, est un mot persan qui signifie couleur. Voir Aךmad 'Abd al-Raziq, al-Runuk, Le Caire, (2001), p. 48. 
personnifiant les sultans, les émirs et les hauts fonctionnaires, pour les distinguer des autres. ${ }^{(1)}$

L'étude des blasons mamluks nous permet de les classer en quatre groupes différents: blasons personnels (privés), blasons fonctionnels (simples et composés), blasons épigraphiques (cartouches) et tam $\Gamma \alpha \mathrm{s}$.

Le blason figurant sur notre flacon appartient au deuxième groupe, constituant un blason composé.

Notons que les blasons composés, formés de plusieurs signets, apparurent pour la première fois sous le règne du sultan al-Zahir Baybars alBunduqdari, mais connurent leur véritable élaboration sous le règne du

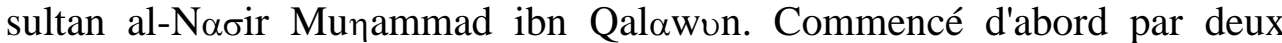
symboles, ils se sont évolués à travers les années pour atteindre enfin le nombre de neuf à l'époque des sultans mamluks circassiens. ${ }^{(2)}$

Notre blason, formé de deux signets, représente le blason personnel du sultan, en plus le blason fonctionnel du propriétaire du flacon dans la cour:

Le médaillon circulaire, renferme la représentation d'un lion (personnifiant ainsi le sultan al-Zahir Baybars ${ }^{(3)}$, au dessus d'une nappe (blason du $\gamma$ ammdar, garde robe ${ }^{(4)}$, faisant ainsi allusion à la fonction de $\breve{S}$ ams al-Din al-Tunbu $\Gamma \alpha$ dans la cour du sultan Baybars.

Le lion, dessiné en réserve, et dirigé vers le gauche, levant sa patte de devant, repose sur une nappe exécutée en émail noir.

(1) Minikae, The Mamluk Heraldry, Notes Given in the Cairo University, Faculty of Archeology, in (1974/ 1975), p. 9; Maysa Dawvd, al-Runvk al-islamiya, Mi $\gamma a l a t$ alDarah, III, (1402/ 1982), p. 27.

(2) Aךmad `Abd al-Raziq, al-Runuk al-isl $\alpha$ miya, pp. 155, 159.

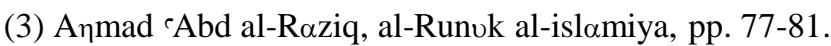

(4) Mayer, Saracenic Heraldry, p. 14. Mayer nous apprend, d'autre part, que le blason de Bahodur al-Hamawi, qui occupa le poste de ra's nawbat al- $\gamma$ ammd $\alpha$ riyah sous le règne

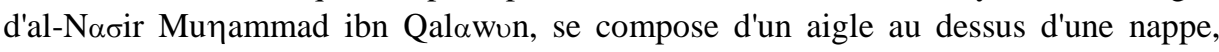
voir Mayer, Saracenic Heraldry, p. 33. 
Nous pouvons donc conclure que les vases en forme sphéro-conique, dont la fonction est restée pour longtemps sujet à controverses, ont servi de qaw $\alpha$ rir de naf $\tau$, flacons à feu grégeois.

Grâce aux textes arabes, nous possédons de preuves évidentes concernant l'usage d'un nombre considérable de flacons de naphte en poterie tout aussi bien en verre.

Les manuscrits de furusiya, à leur tour, nous ont été d'une grande utilité: ils nous ont fourni de détails remarquables à propos des différents types de flacons, ainsi, il en était de forme globulaire ou ovoïde, de taille moyenne ou grande, en poterie ou en verre.

D'autre part, l'étude nous a permis de distinguer une nouvelle fonction de flacons à feu grégeois: outre la projection, ils ont servi de "containers" de naphte destiné à l'arrosage des flèches incendiaires lors des batailles, permettant ainsi un envisagement meilleur du décor .

Il ressort de l'étude, que le flacon du musée d'art islamique du Caire

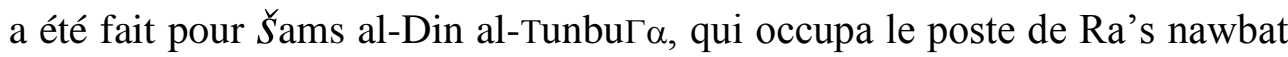
al- $\gamma$ ammdariyah, au cours du règne du sultan al-Zahir Baybars alBunduqdari (658-676 H. /1260-1277).

D'après les historiens, il aurait été assassiné par ordre du sultan alNaбir Munammad ibn Qalawon, suite à l'assassinat d'al-Ašraf Halil ibn Qalawon, ce qui nous a permis de dater le flacon vers 693 H. /1293-1294.

Le décor du flacon représente une inscription à titulature, des motifs floraux et animaliers ainsi que les blasons du commanditaire. 


\section{Description des planches}

Pls. I-VI: Flacon de naphte en verre émaillé, au nom de l'émir šams al-Din

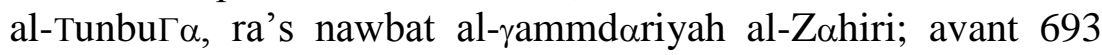
H./ 1293-1294 Musée d'art islamique du Caire, no. 18038.

PI. VII: Un flacon de naphte en verre émaillé au nom du sultan rasulide alAšraf cUmar ibn Yusuf (694-696 H./ 1295-1296) conservé au musée du Louvre-

PI. VIII: Flacon en verre manganèse au nom du sultan rasulide al-Ašraf Umar ibn Yusuf (694-696 H./ 1295-1296), conservé au musée de Victoria et Albert-d'après Gilded and Enamelled Glass.

PI. IX: Flacon de naphte en poterie sans glaçure, remontant à l'époque fatimide, Ve /XIe siècle-d'après la Variété dans l'unité.

PI. X: Flacon de naphte en poterie sans glaçure, VIe /XIIe siècle, conservé au musée d'art islamique du Caire, no. 2330.

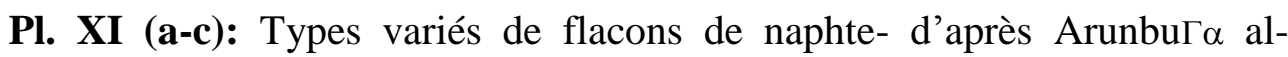
Zardak $\alpha \breve{s}$

PI. XII: flacons de naphte en forme conique, et sphéro-conique-dans Kit $\alpha \mathrm{b}$ al-mahzun fi $\gamma \alpha \mathrm{mi}^{\ulcorner}$al-funun-d'après Pages of Perfection

PI. XIII: Un lanceur de naphte en train de lancer son flacon dans-Kitab al-

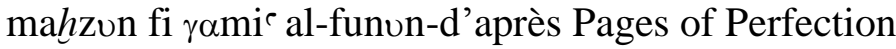

PI. XIV: Un groupe de lanceurs de naphte, naff $\alpha \tau i n$, aux vêtements

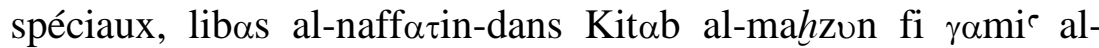
funun-d'après Pages of Perfection. 


\section{Bibliogrqphie}

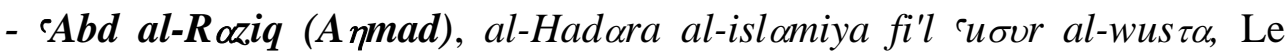
Caire, (1990).

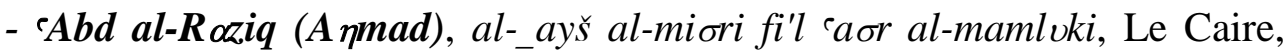
(2000).

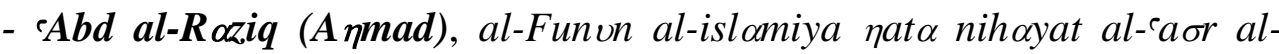
fatimi, Le Caire, (2001).

- ‘Abd al-Roziq (A qmad), al-Runvk al-islomiya, Le Caire, (2001).

- ‘Abd al-Roziq (A nmad), al-Funon al-islomiya fi'l 'a orayn al-ayyobi wa'l mamlvki, Le Caire, (2006).

- 'Abd al-`Aziz, (Wis om), "al-Nor al-IIriquiyah, tabieat tarkibiha wa atoriha

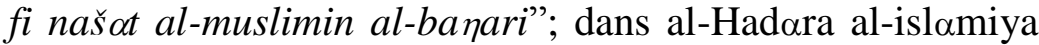
wa 'alam al-binar, colloque organisé par la Fédération des historiens arabes, Le Caire, 6-8 Novembre, (1993).

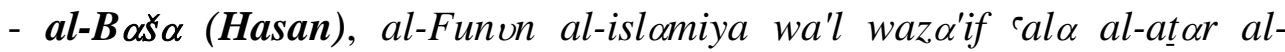
carabiya, Le Caire, (1966-1967).

- al-Dimašqay, šadarat al-dahab fi ahbor man dahab, Beyrut, al-Maqdisi (šihab al-Din), Kitab al-Rawdatayn fi ahbor al-dawlatayn alnuriya wa'l oal aniya, Réalisé par Ibrahim al-Zaybaq, Beyrut, (1997)

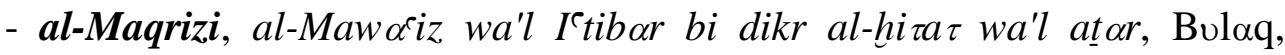
(1853).

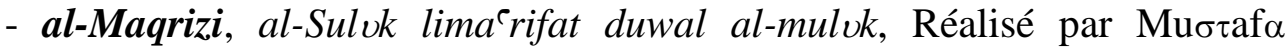
Ziyada, Le Caire, (1936).

- al-Nuwayri, Nihoyat al-arab fi funvn al-adab, XXIX,

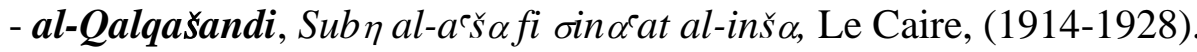

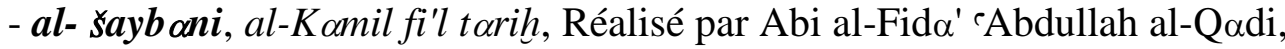
Beyrut, (1415 H./ 1995).

- al-Suyvii, Torih al- hulafo', Réalisé par Munyi al-Din ‘Abd al-Hamid, Le Caire, (1371 H./ 1952). 
- al-Tabari, Torih al-umam wa'l mulvk, Bayrut, (1407 H).

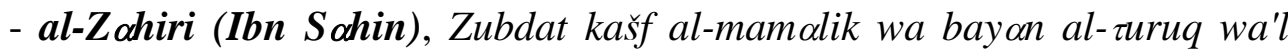
mas alik, Paris, (1894).

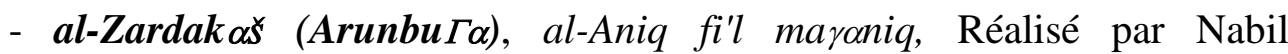
Mūammad ‘Abd al- ‘Aziz, Le Caire, (1981).

- Ayalon (David), Gunpowder and Fire Arms in the Mamluk Kingdom, London, (1978).

- Bahgat (Ali) et Massoul (Félix), La céramique musulmane de l'Egypte, Le Caire, (1930).

- Cahen (Claude), Un traité d'armurerie composé pour Saladin, Bulletin d'Etudes Orientales, XII, Beyrut, (1948).

- Chester (Greville J.), "On the Pottery and Glass Found in the Excavations", The Recovery of Jerusalem, A Narrative of Exploration and Discovery in the City and the Holy Land, London, (1871).

- Dawud (Moysa), al-Runvk al-islomiya, Miralat al-Darah, III, (1402/ 1982).

- Dozy, Supplément aux dictionnaires arabes, Leyde, (1881).

- Ettinghausen (R.), The Uses of Sphero-Conical Vessels in the Muslim East, Journal of Near Eastern Studies, Chicago, XXIV, (1965).

- Fehérvari (Géza), Ceramics of the Islamic World, in the Tareq Ragab Museum, London.

- Freestone (C.) et Stapleton (Colleen P.), Composition and Technology of Islamic Enamelled Glass of the Thirteen and Fourteen Centuries, dans Gilded and Enamelled Glass from the Middle East, Edited by Rachel Ward, London, (1998).

- Goulchani (A.) and Adle (C.), A Sphero-Conical Vessel as Fuqa'a or a Gourd for "Beer", Muqarnas, 9, (1992).

- Hildburgh (W. L.), “Aeolipiles as Fire Blowers”, Archaeologia, (Oxford), XCIV, (1951). 


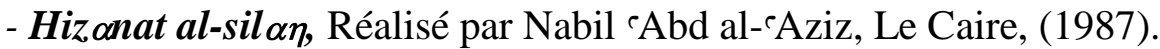

- Ibn al-_awzi, al-Muntazim min 257 H., Beyrut, (1358 H).

- Ibn Hajar al-eAsqaloni, al-Durar al-kominah fi a ciyon al-ma'ah altomina, Réalisé par Munammad Han, Hidrabad, (1972).

- Ibn Katir, al-Bidoyah wa'l nihoyah, Beyrut.

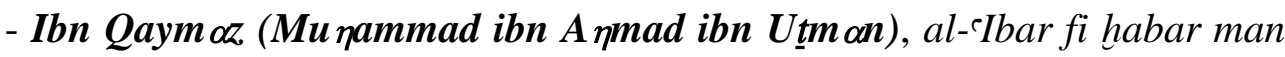

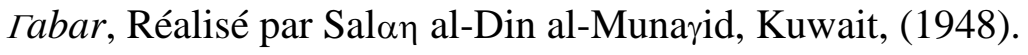

- Ibn Qaymoz. (Munammad ibn A pmad ibn Utmon), Siyar aclom al-

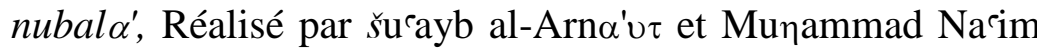
al--Irqsusi, Beyrut, (1413 H).

- Ibn Ta Tribirdi, al-Nuyom al-Zahira fi mulok mior wa'l qahira, Le Caire, (1930-1972).

- Kuhnel (Ernest), Islamic Arts, London, (1963).

- Lane, Early Islamic Pottery, London, (1947).

- Majid (‘Abd al-Muncim), Nuzum dawlat salatin al-mamalik wa rus umihim fi mior, Le Caire, (1967).

- Marzoq (Mu nammad ‘Abd al-`Aziz), al-Funon al-zuhrufiya al-islomiya fi mior qabl al-fatimiyyin, Le Caire, (1974).

- Mayer (L. A.), Saracenic Heraldry, A Survey, Oxford, (1933).

- Mercier (M.), Le feu grégeois, les feux de guerre depuis l'antiquité, la poudre à canon, Paris, (1952).

- Minikae (M.), The Mamluk Heraldry, Notes Given in the Cairo University, Faculty of Archeology, in (1974-1975).

- Mustafa (Muhammad), "Neuerwerbungen des Museums fur Islamisch Kunst inn Kairo" in Aus der Welt der islamischen Kunst, Festscrift fur Ernst Kuhnel, Berlin, (1959).

- Pages of Perfection, Islamic Painting and Calligraphy from the Russian Academy of Sciencesm St. Petersbergm Lugqnom (1995). 
- Pradines (Stéphane), Michaudel (Benjamin) et Monchamp (Julie), La muraille ayyubide du Caire: les fouilles archéologiques de bab al-barqiya et bab al-manruq, AnIsl. , XXXVI, Le Caire, (2002).

- Qaddoumi (Ghada), La variété dans l'unité, Dor al-ațar al-islamiya, Kuwait, (1987).

- Quatremère, Histoire des sultans mamlouks, (1845).

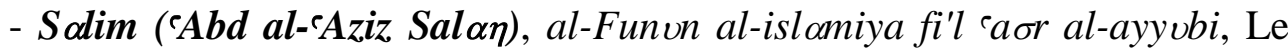
Caire (2000).

- Sauvaget, Flacons à vin ou grenades à feu grégeois? Dans Mélanges Grégoire (Annuaire de l'Institut de Philologie et d'Histoire Orientales et Slaves), IX, Bruxelles, (1949).

- Seyrig (Henri), Flacons? Grenades? Eolipiles? Syria, XXXVI, Paris, (1959).

- Speel (Erika), Dictionary of Enamelling, (1998).

- Yusuf ('Abd al-Ra'vf eAli), Dirosa fi'l fuhor al-miori, qaworir al-naf, dirasat atariya islamiya, I, Le Caire, (1978).

- Yusuf ('Abd al-Ra' of 'Ali), Syro Egyptian Glass, Pottery and Wooden Vessels in Gilded and Enamelled Glass in the Middle East, London, (1998), pp. 20-23. 


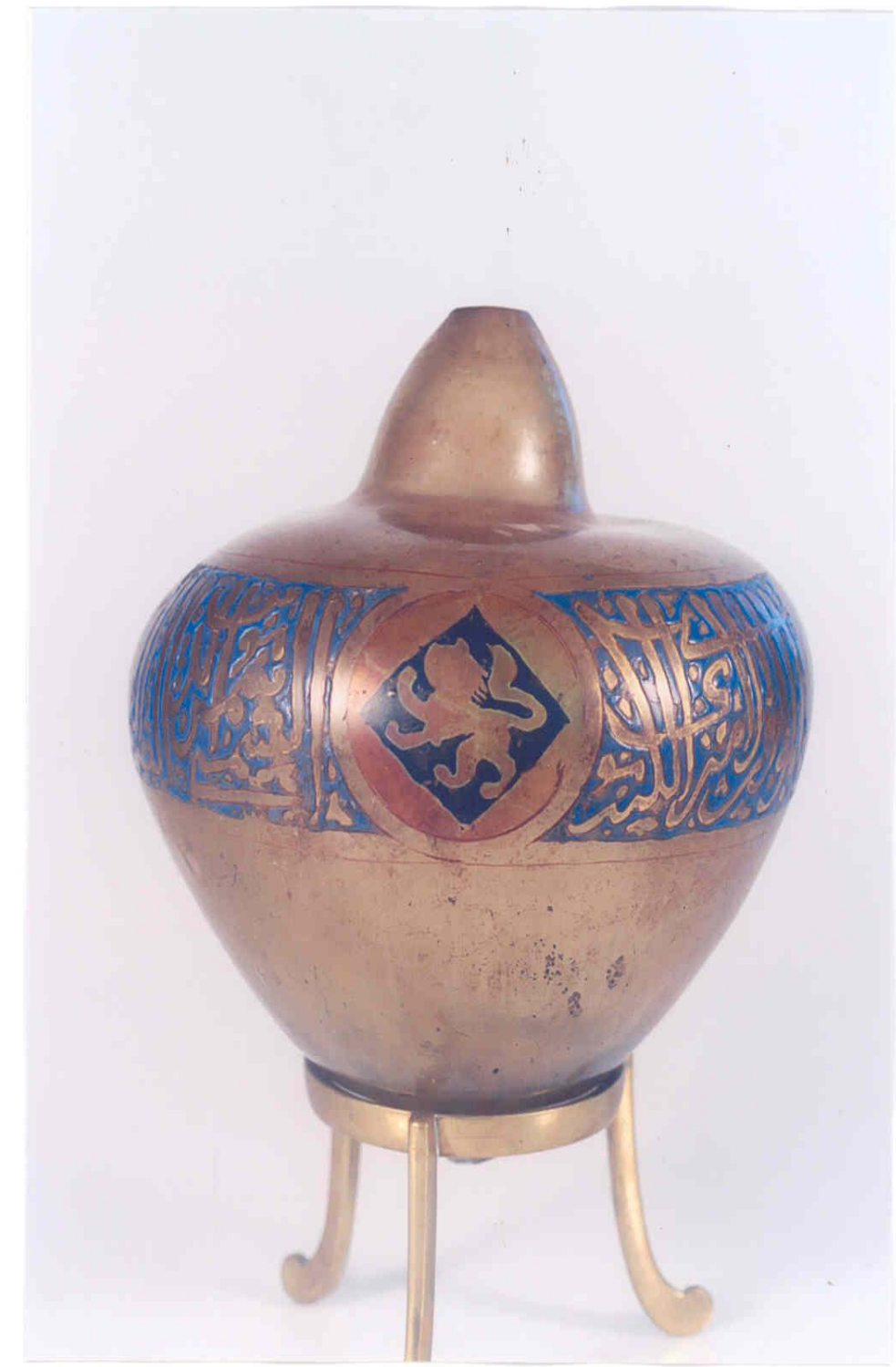

PI. I 
صفحة فارغة 


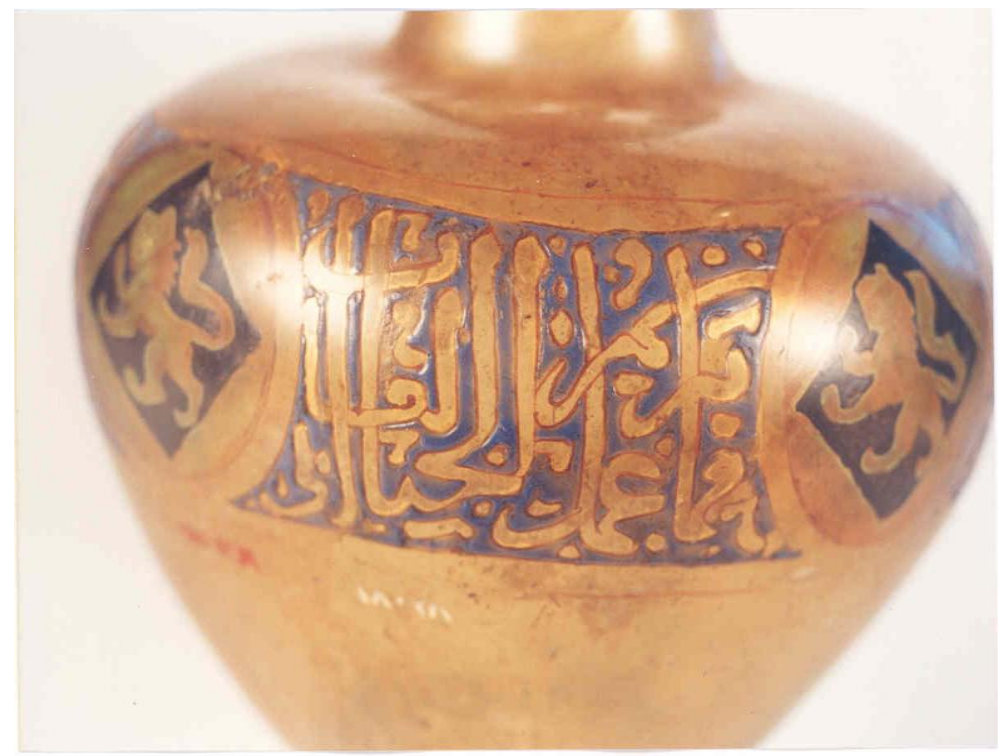

PI. II

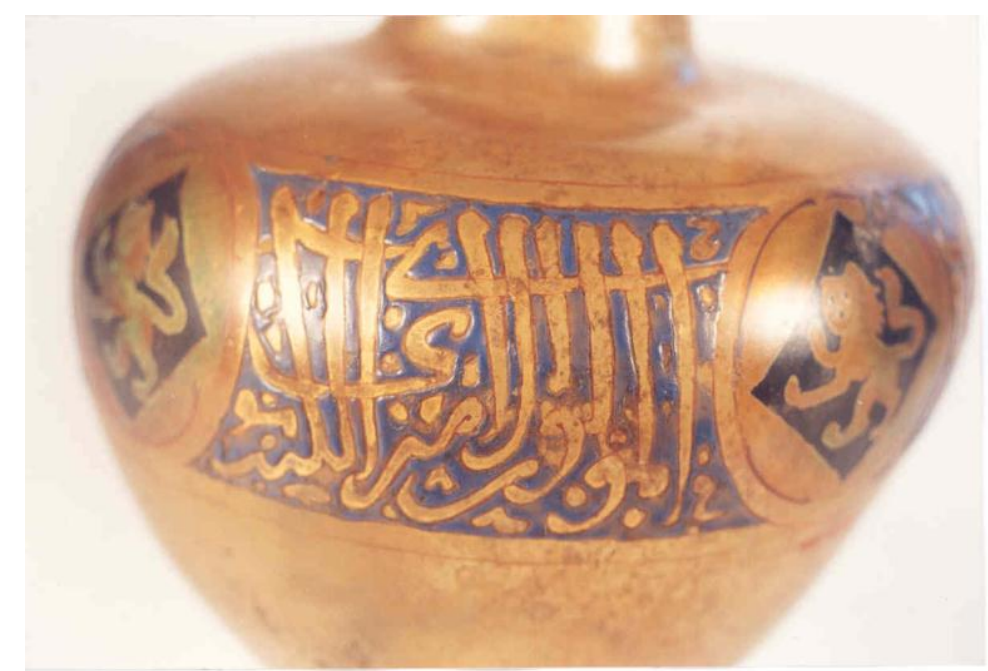

PI. III 


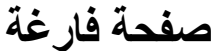




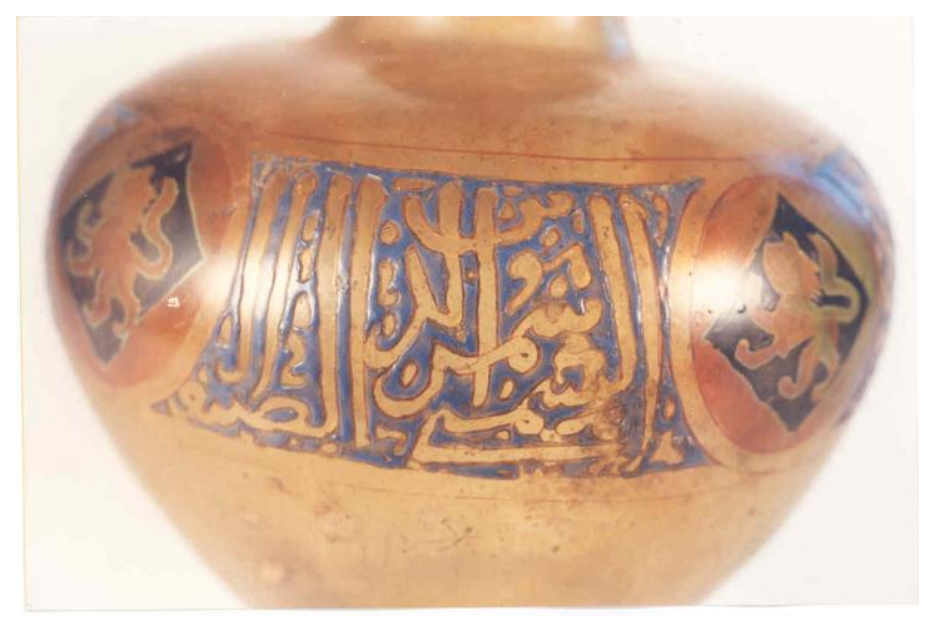

PI. IV

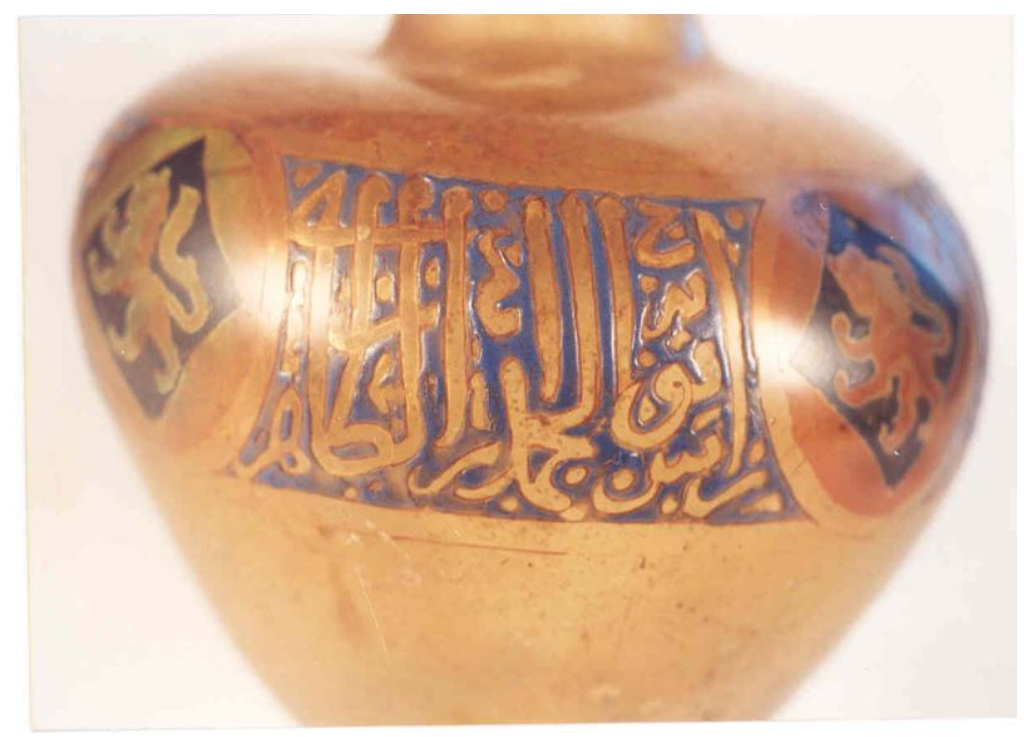

PI. V 


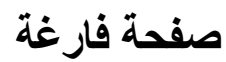




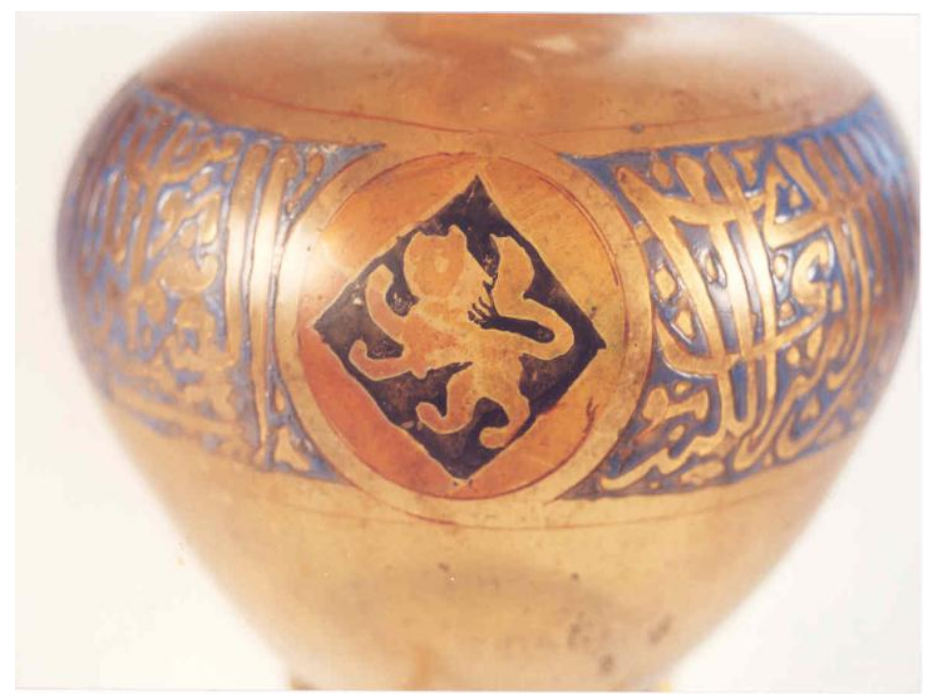

PI. VI

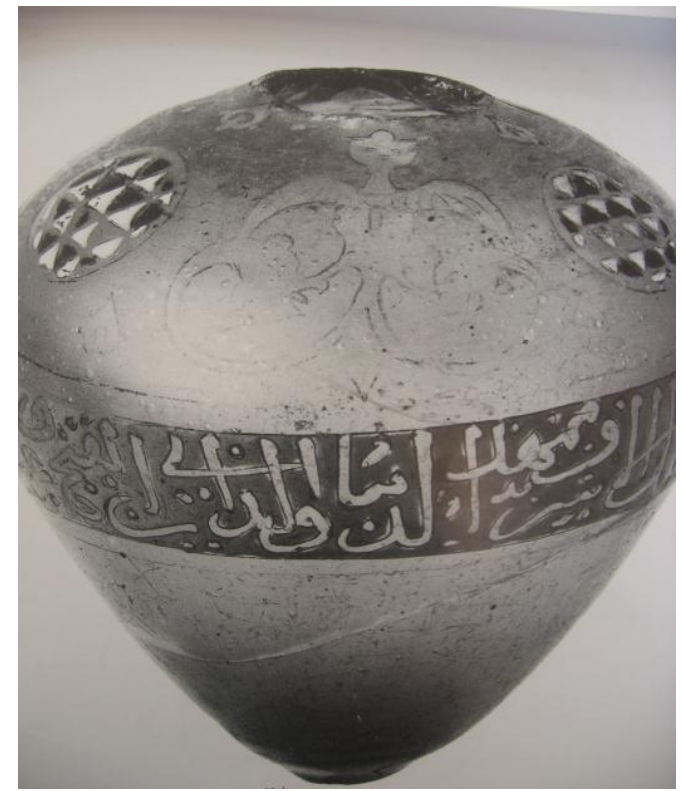

PI. VII 


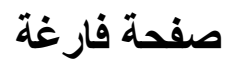




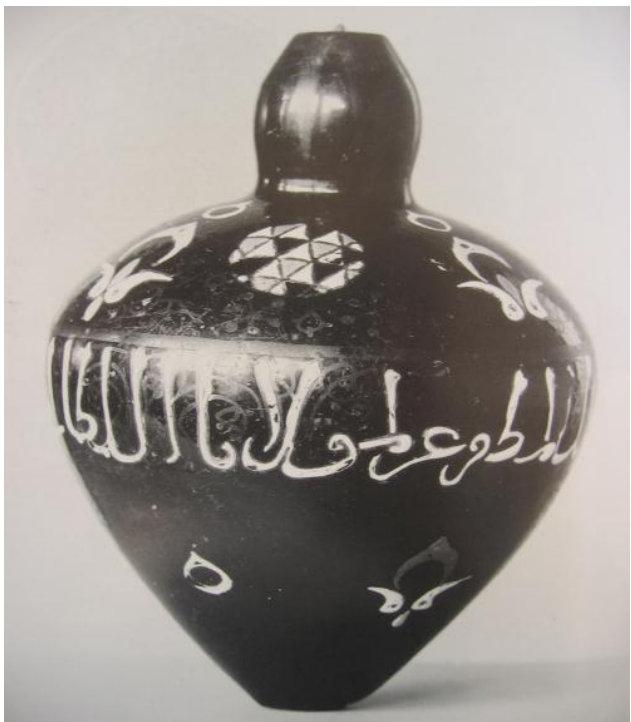

PI. VIII

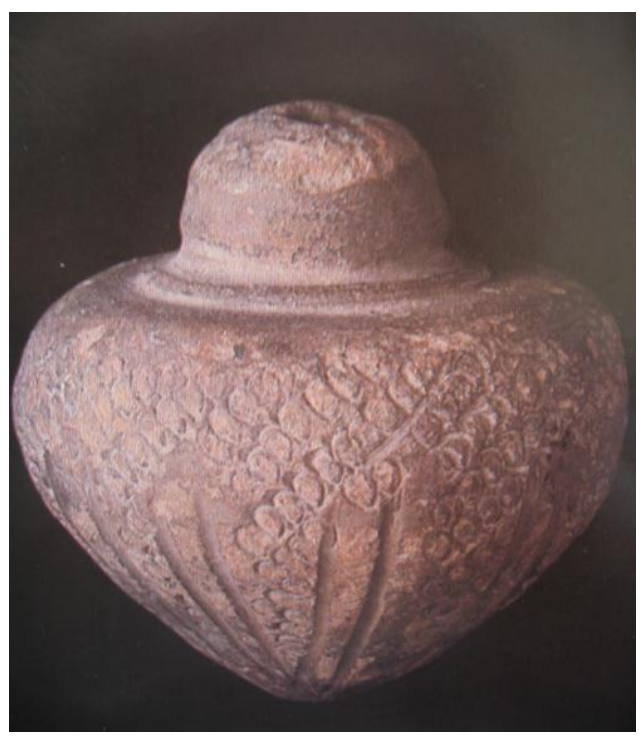

PI. IX 


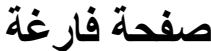




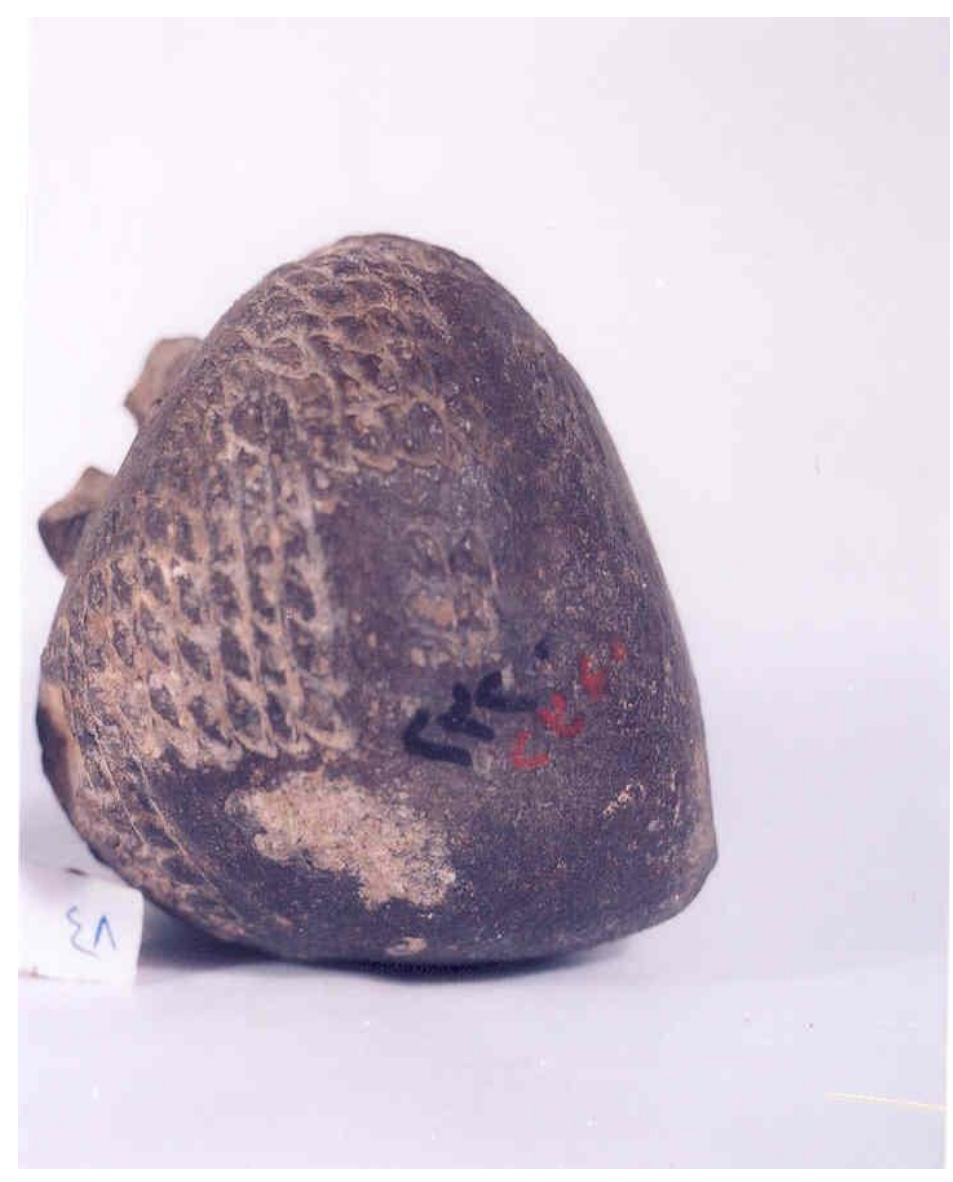

PI. X 
صفحة فارغة 


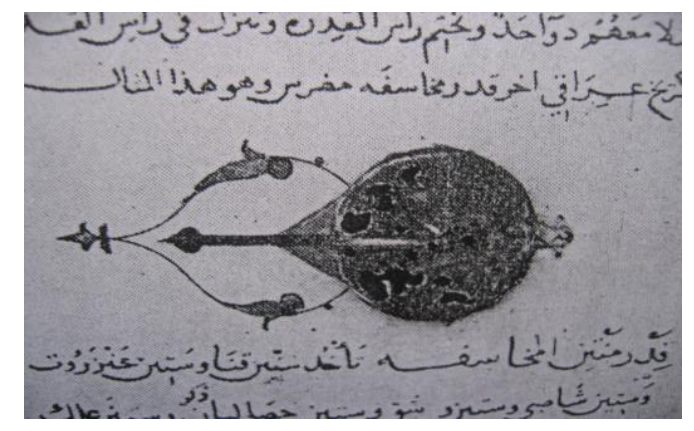

a

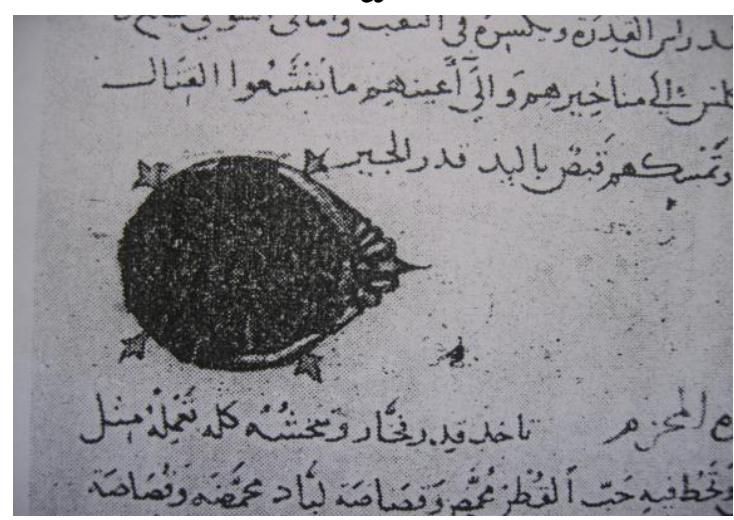

b

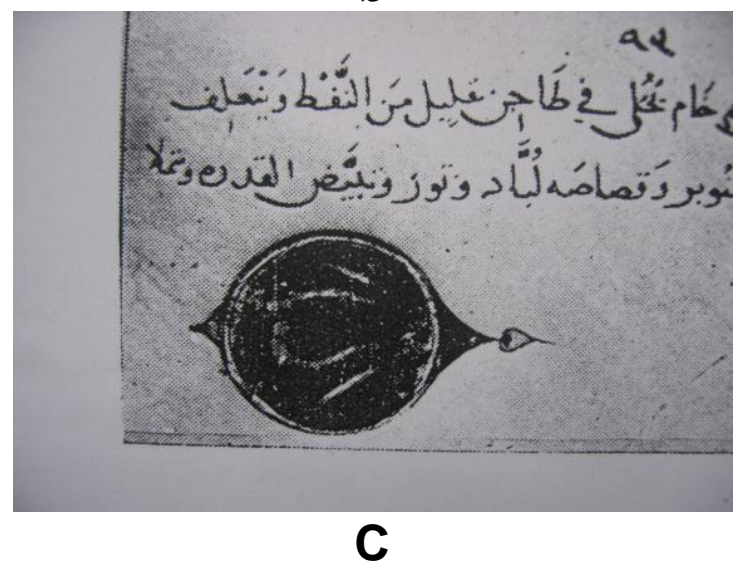

PI. XI (a-c) 


\section{صفحة فارغة}




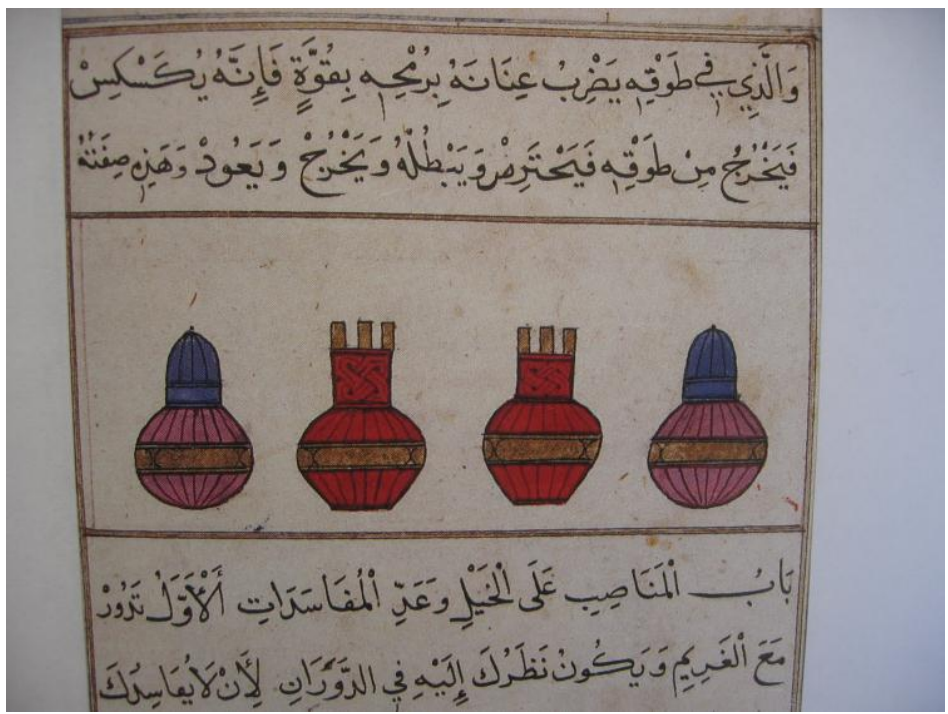

PI. XII

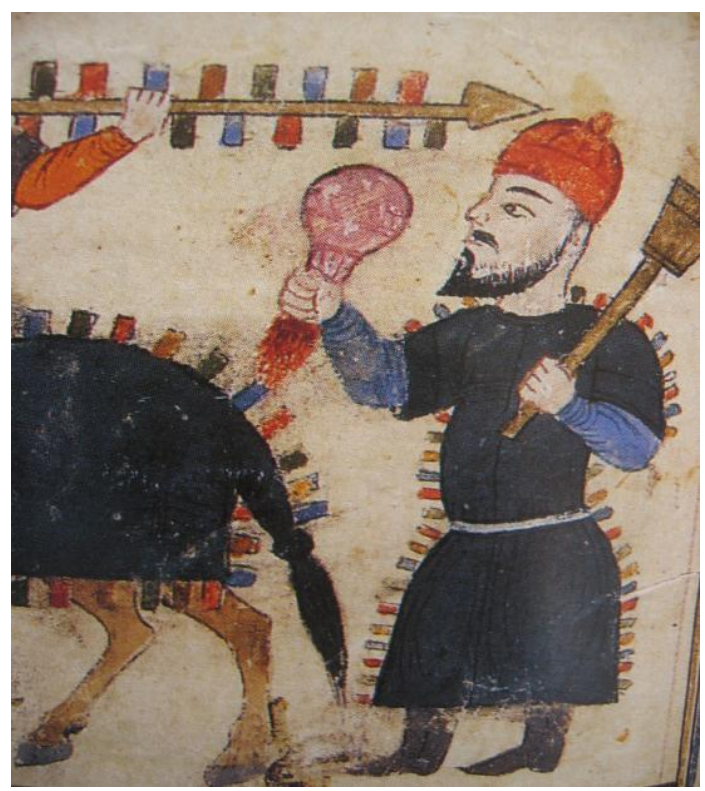

PI. XIII 


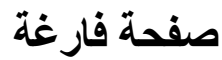




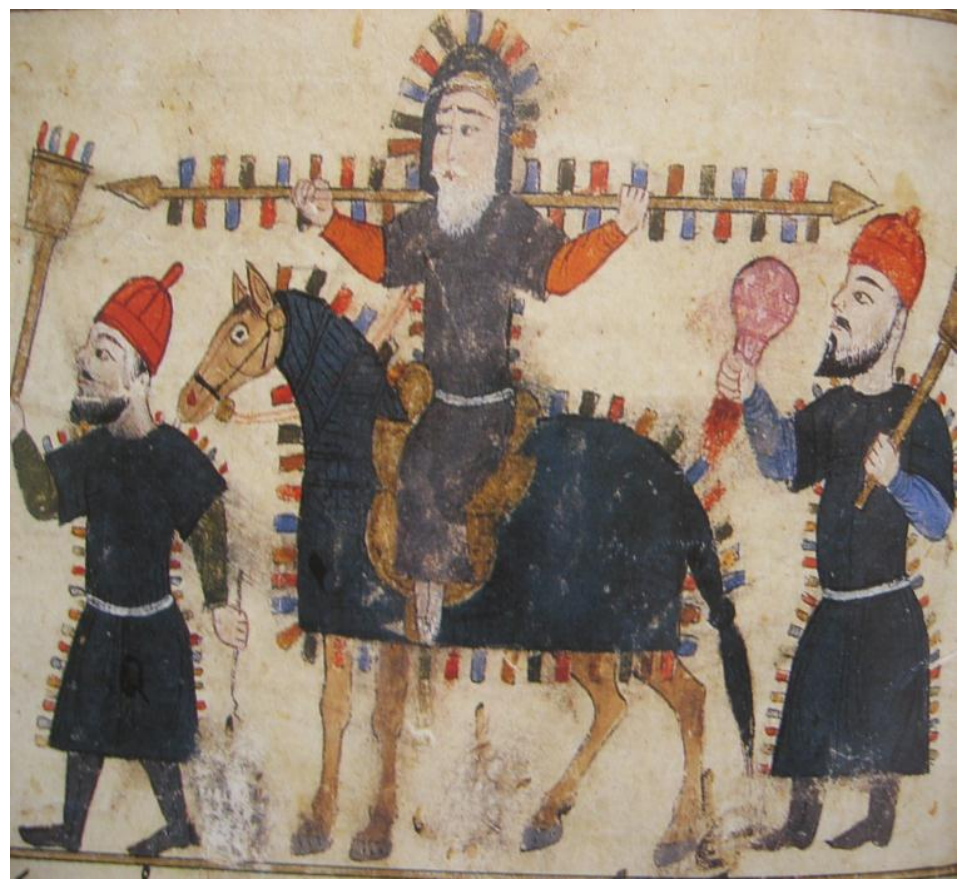

PI. XIV 